\title{
Uniformly hyperbolic surfaces
}

\author{
Mario Bonk* and Alexandre Eremenko ${ }^{\dagger}$
}

February 29, 2000

\begin{abstract}
We prove the equivalence of certain uniform hyperbolicity conditions for open simply connected surfaces with non-positively curved intrinsic metric.
\end{abstract}

\section{Introduction}

We consider open simply connected surfaces (two-dimensional topological manifolds) equipped with intrinsic metrics. This means that the distance between two points is equal to the infimum of the lengths of curves connecting these points. An additional requirement is that our surfaces are "surfaces of bounded curvature" in the sense of A.D. Aleksandrov [3, 16], which we call Aleksandrov surfaces. Before stating the formal definition, we mention the two most important special cases:

A. Surfaces with smooth Riemannian metrics, and

B. Surfaces with polyhedral metrics, which means that every point of such a surface has a neighborhood isometric to a cone, that is, the unit disc $\mathbf{D}$ with the length element

$$
c|z|^{\alpha-1}|d z|, \quad c>0, \alpha>0 .
$$

Surfaces as in B arise in the theory of analytic functions as "simply connected Riemann surfaces spread over the plane". If $g$ is analytic in a disc $D(R):=\{z:|z|<R\}, R \leq \infty$, the surface $S_{g}$, obtained by equipping $D(R)$

*supported by a Heisenberg fellowship of the DFG.

${ }^{\dagger}$ supported by a grant from the NSF. 
with the length element $\left|g^{\prime}(z)\right||d z|$, is a surface with polyhedral metric. In this case $\alpha$ is always an integer.

Aleksandrov surfaces constitute a natural class of metric spaces which contains the classes $\mathrm{A}$ and $\mathrm{B}$, and is closed with respect to certain limit processes.

Of the several equivalent definitions of Aleksandrov surfaces we here state the simplest one; an intrinsic definition will be given in the next section. An Aleksandrov surface is a topological surface with an intrinsic metric whose length element can be locally defined by

$$
e^{u(w)}|d w|
$$

where $w$ is a complex local coordinate and $u$ is a difference of two subharmonic functions such that $\exp u$ is locally integrable on rectifiable curves in the $w$-plane. The generalized Laplacian $-\Delta u$ is a signed Borel measure $\omega$ on the surface, which is called integral curvature. We say that the surface is non-positively curved if $\omega \leq 0$, that is, all functions $u$ in (1) are subharmonic. In case A above, this is equivalent to the non-positivity of the Gaussian curvature. In case B the integral curvature is a discrete measure with atoms of mass $-2 \pi(\alpha-1)$, so the surface is non-positively curved if $\alpha \geq 1$ everywhere. In particular, if $S=S_{g}$ for an analytic function $g$, then $S_{g}$ is non-positively curved and the integral curvature consists of atoms of mass $-2 \pi\left(\operatorname{deg}_{g}(p)-1\right)$ located at the critical points $p$ of $g$. Here $\operatorname{deg}_{g}(p)$ is the local degree of $g$ at $p$.

A local coordinate $w$, for which (1) holds, is called an isothermal coordinate. Isothermal coordinates define a complex analytic structure on every Aleksandrov surface [12]. Thus we can speak of holomorphic functions, conformal maps, moduli of curve families etc. on an Aleksandrov surface.

By the Uniformization Theorem of Huber [12], every open simply connected Aleksandrov surface is isometric to a disc $D(R):=\{w:|w|<$ $R\}, \quad R \in(0, \infty]$, equipped with a length element $(1)$, where $u$ is a difference of two subharmonic functions in $D(R)$. If $R<\infty$, the surface $S$ is called hyperbolic.

We prove the following result.

Theorem Let $S$ be an open simply connected non-positively curved Aleksandrov surface. Then the following conditions are equivalent:

(i) There exist $R_{0}>0$ and $\epsilon>0$ such that every relatively compact open disc $B\left(a, R_{0}\right) \subset S$ has integral curvature less than $-\epsilon$. 
(ii) $S$ is hyperbolic in the sense of Gromov, that is, there exists a constant $\delta \geq 0$ such that for all points $x, y, z, w \in S$

$$
(x, z)_{w} \geq \min \left\{(x, y)_{w},(y, z)_{w}\right\}-\delta,
$$

where $(u, v)_{w}:=(1 / 2)\{\rho(u, w)+\rho(v, w)-\rho(u, v)\}$, and $\rho$ stands for the intrinsic distance.

(iii) A linear isoperimetric inequality holds on $S$, that is, there exists a constant $C_{1}>0$ such that all Jordan regions $\Omega \subset S$ satisfy

$$
A(\Omega) \leq C_{1} \ell(\partial \Omega),
$$

where $A$ stands for the area and $\ell$ for the length.

(iv) $S$ is tight, that is, there exists a constant $C_{2}>0$ such that for all holomorphic maps $f: \mathbf{D} \rightarrow S$ we have $\left\|f^{\prime}(z)\right\| \leq C_{2}$ for $z \in \mathbf{D}$. Here the norm $\left\|f^{\prime}(z)\right\|$ of the derivative is the ratio at $z$ of the pull-back of the length element on $S$ to the length element of the Poincaré metric of constant negative curvature -1 on $\mathbf{D}$.

The proof of the Theorem will show that the implications between these conditions are quantitative, that is, the parameters in (i)-(iv) only depend on each other. We emphasize that we do not assume $S$ to be complete. This will cause some complications in the proof.

Condition (i) is a generalization of the condition of Bloch's Theorem $[5,1]$. For example, if $S$ is a plane region with Euclidean metric, then (i) is equivalent to boundedness of the inner radius. Bloch's Theorem establishes the implication (i) $\Rightarrow$ (iv) for the case $S=S_{g}$, where $g$ is a holomorphic function in the unit disc.

Condition (ii) was introduced in the theory of groups [10]. It defines a notion of hyperbolicity for arbitrary metric spaces. Our proof of the implication (ii) $\Rightarrow$ (iii) follows [10, pp. 96-97]. Since a non-complete surface $S$ need not be a geodesic space, some modifications are necessary.

The linear isoperimetric inequality (iii) probably occurred for the first time is Ahlfors's "Theorie der Überlagerungsflächen" [2]. The implication (iii) $\Rightarrow$ (iv) for the case $S=S_{g}$ is close to the results of Dufresnoy [9] and Hayman [11, Ch. VIII] (Ahlfors, Dufresnoy and Hayman used the spherical metric).

The tightness condition (iv) in the case $S=S_{g}$ is related to the uniform normality conditions in [11]. If $S_{g}$ is tight, $g$ is usually called a Bloch function 
$[14,15]$. The tightness condition was also studied in the theory of hyperbolic complex spaces in higher dimensions [13]. The implication (iv) $\Rightarrow$ (i) in the case $S=S_{g}$ is a simple consequence from Schwarz's Lemma.

Our implication (i) $\Rightarrow$ (iv) may be considered as a generalization of the Ahlfors-Schwarz Lemma. In this lemma a pointwise negative upper bound for the Gaussian curvature of a conformal metric on the disc implies an upper bound for the density of the metric in terms of the Poincaré density. Our Theorem gives such a bound under weaker assumptions on the metric.

The implication (iv) $\Rightarrow$ (iii) yields the following inequality: for every logarithmically subharmonic function $g$ in $\mathbf{D}$ satisfying

$$
g(z) \leq C_{3}\left(1-|z|^{2}\right)^{-1} \quad \text { for } \quad|z|<1,
$$

we have

$$
\int_{|z| \leq r} g(z)^{2} d m(z) \leq C_{4} \int_{|z|=r} g(z)|d z|,
$$

where $d m$ is the area element, and $C_{4}$ depends only on $C_{3}$.

In particular, this inequality holds for $g=\left|f^{\prime}\right|$, where $f$ is a Bloch function. If $h$ is a univalent function in the unit disc, then $g=h^{\prime \prime} / h^{\prime}$ satisfies (3) with $C_{3}=6[14,15]$. So we obtain the following inequality for univalent functions

$$
\int_{|z| \leq r}\left|\frac{h^{\prime \prime}(z)}{h^{\prime}(z)}\right|^{2} d m(z) \leq C_{5} \int_{|z|=r}\left|\frac{h^{\prime \prime}(z)}{h^{\prime}(z)}\right||d z|,
$$

where $C_{5}$ is an absolute constant. After we communicated this inequality to Kari Astala, he gave a very short proof, based on the $L^{1}$ estimate of the non-tangential maximal function.

We ask whether the assumption of non-positive curvature in our Theorem can be replaced by the weaker assumption that the Gaussian curvature is bounded from above (appropriate modification of the conditions may be necessary for this). B. Kleiner informed us that he proved the equivalence of conditions (iii) and (iv) under this weaker assumption. Also, the referee of this paper noticed that our arguments permit replacing the condition of non-positive curvature by the slightly weaker condition,

$$
\omega^{+}(S) \leq C<\pi
$$

where $\omega^{+}$is the positive part of the integral curvature.

In the next section we state the necessary facts from Aleksandrov's theory. The reader that is interested in surfaces as in case A or B only, may skim through this section. 


\section{Aleksandrov surfaces}

In this section $S$ stands for an open simply connected surface with an intrinsic metric $\rho$. The closure of a Jordan region will be called a closed Jordan region. It is homeomorphic to the closed unit disc. The boundary of a Jordan region is always oriented so that the region is on the left.

Curves will always be oriented. A curve is called a shortest curve if its length is equal to the distance between its endpoints. If $x$ and $y$ are two points in $S$, then by $[x, y]$ we denote a shortest curve from $x$ to $y$, if it exists. This notation is ambiguous, because we do not require uniqueness of $[x, y]$. Evidently all shortest curves are simple arcs. A subarc of a shortest curve is also a shortest curve. If $x$ and $y$ are two points on a simple arc $L$, then we denote by $L[x, y]$ the subarc of $L$ from $x$ to $y$.

A broken line is a curve $L$ that can be decomposed into a sequence of shortest curves, that is, there are points $x_{0}, \ldots, x_{n} \in L$ such that $L=$ $\left[x_{0}, x_{1}\right] \cup \ldots \cup\left[x_{n-1}, x_{n}\right]$.

Suppose $L$ and $M$ are simple curves with a common endpoint $O$. Let $x \in L, y \in M, x, y \neq O$, and consider a Euclidean triangle with sides of lengths $\rho(x, O), \rho(y, O), \rho(x, y)$. Let $\gamma(x, y) \in[0, \pi]$ be the angle opposite the side whose length is $\rho(x, y)$. The upper angle between $L$ and $M$ is defined as

$$
\overline{\angle(L, M)}=\limsup _{x, y \rightarrow O} \gamma(x, y) \in[0, \pi] .
$$

Here the points $x \in L$ and $y \in M$ tend to $O$ in the sense of the parameterizations of the curves $L$ and $M$. If the limit exists it is called the angle between $L$ and $M$ and denoted by $\angle(L, M)$. We warn the reader that conformal maps, as defined in the Introduction, may not preserve angles at some points. If $L \cup M$ is a shortest curve containing $O$ as an interior point, then $\angle(L, M)=\pi$.

A geodesic triangle is a closed curve of the form $\left[x_{1}, x_{2}\right] \cup\left[x_{2}, x_{3}\right] \cup\left[x_{3}, x_{1}\right]$. A simple triangle $\Delta$ is a closed Jordan region whose oriented boundary $\partial \Delta$ consists of three shortest curves $L_{1}=\left[x_{1}, x_{2}\right], L_{2}=\left[x_{2}, x_{3}\right], L_{3}=\left[x_{3}, x_{1}\right]$, and has the additional property that for any two points $x, y \in \partial \Delta$ there exists a shortest curve $[x, y] \subset \Delta$. The upper excess $\bar{\delta}(\Delta)$ of the simple triangle $\Delta$ is defined as

$$
\bar{\delta}(\Delta)=\overline{\angle\left(L_{1}, L_{2}\right)}+\overline{\angle\left(L_{2}, L_{3}\right)}+\overline{\angle\left(L_{3}, L_{1}\right)}-\pi .
$$

Two sets are said to be non-overlapping if they have disjoint interior. 
A surface $S$ with intrinsic metric is called an Aleksandrov surface if for every point in $S$ there exists a neighborhood $U$, homeomorphic to the open unit disc $\mathbf{D}$, and a constant $C(U)<\infty$ such that for any system $T_{1}, \ldots, T_{n}$ of non-overlapping simple triangles contained in $U$ we have the inequality

$$
\sum_{i=1}^{n} \bar{\delta}\left(T_{i}\right) \leq C(U) .
$$

According to Reshetnyak [16] and Huber [12], this geometric definition is equivalent to the analytic definition stated in the Introduction.

In the following we assume that $S$ is an open simply connected Aleksandrov surface. Every point in an Aleksandrov surface has arbitrarily small convex neighborhoods $U$. This means that every two points in $U$ can be connected by a shortest curve, which belongs to $U$.

A fundamental result of Aleksandrov and Zalgaller says that for every shortest curves $L$ and $M$ with a common endpoint $O$ the angle $\angle(L, M)$ exists (see [3, p. 116] or [16, Theorem 8.2.3]). If $L$ and $M$ have only the point $O$ in common, then every small neighborhood of $O$ is divided by $L \cup M$ into two components. The closures $F$ and $F^{\prime}$ of these components are called sectors with vertex $O$ determined by $L$ and $M$. We say that $F$ and $F^{\prime}$ are complementary at $O$. We suppose that $L$ is oriented towards $O$ and $M$ from $O$. Then one of the two complementary sectors, say $F$ is on the left of the oriented curve $L \cup M$, and the other $F^{\prime}$ is on the right.

The sectorial angle $\angle_{s} F \in[0, \infty)$ of a sector $F$ is defined as the least upper bound of all sums

$$
\sum_{k=1}^{n} \angle\left(L_{k}, M_{k}\right),
$$

where $L_{k}$ and $M_{k}$ are shortest curves with endpoint $O$ forming non-overlapping sectors $F_{k} \subset F$. Two sectors determined by the same curves $L$ and $M$ and lying on the same side of $L \cup M$ have the same sectorial angle. If $F$ and $F^{\prime}$ are complementary sectors at $O$, then the sum $\angle_{s} F+\angle{ }_{s} F^{\prime}$ only depends on $O$ and is called the total angle $\theta(O)$ at $O$. We have $\theta(O)=2 \pi-\omega(\{O\})$, where $\omega$ is the integral curvature.

Using this terminology, a version of the Gauss-Bonnet Theorem can be stated as follows.

Proposition 1 Let $\Omega \subset S$ be a Jordan region bounded by a Jordan broken line $\partial \Omega=L_{0} \cup \ldots \cup L_{n-1}$, where $L_{j}$ are shortest curves, labeled by residues 
modulo $n$, in the natural cyclic order on $\partial \Omega$. Denote by $\alpha_{k}$ the sectorial angle of the sector defined by $L_{k-1}$ and $L_{k}$, which is contained in $\bar{\Omega}$. Then

$$
\omega(\Omega)+\sum_{k=1}^{n} \tau\left(L_{k}\right)+\sum_{k=1}^{n}\left(\pi-\alpha_{k}\right)=2 \pi,
$$

where $\tau\left(L_{k}\right) \leq 0$.

In this proposition $\tau\left(L_{k}\right)$ is the so-called left turn of the shortest curve $L_{k}$, [3, cf. pp. 199, 214, 215]; we only need the fact that $\tau(L) \leq 0$ for every shortest curve $L$.

Proposition 2 If $F$ is a sector formed by shortest curves $L$ and $M$ meeting at $O$, and $\angle_{s} F<\pi$, then there is a shortest curve $\Gamma$ in $F$, connecting a point $x \in L$ to a point $y \in M$ such that

$$
\ell(\Gamma)<\ell(L[x, O])+\ell(M[O, y]) .
$$

Outline of proof. We will apply this proposition only to non-positively curved Aleksandrov surfaces, so we discuss the proof only for this case.

Let $F^{\prime}$ be the sector complementary to $F$. Since $S$ is non-positively curved, we have

$$
\angle{ }_{s} F+\angle{ }_{s} F^{\prime}=\theta(O)=2 \pi-\omega(\{O\}) \geq 2 \pi .
$$

Hence $\angle{ }_{s} F^{\prime}>\pi$.

Since $\angle(L, M) \leq{ }_{s} F<\pi$ by the definition of sectorial angle and our assumption, the definition of angle shows that

$$
\rho(x, y)<\rho(x, O)+\rho(O, x),
$$

if $x \in L$ and $y \in M, x, y \neq O$, are sufficiently close to $O$.

Moreover, for these points $x$ and $y$, we may assume that there exists a geodesic $K=[x, y]$. Let $x^{\prime}$ be the last point of $K$ on $L$ and $y^{\prime}$ the first point of $K$ on $M$. Then $K\left[x^{\prime}, y^{\prime}\right]$ is completely contained in $F$ or in $F^{\prime}$. Hence $L\left[x, x^{\prime}\right] \cup K\left[x^{\prime}, y^{\prime}\right] \cup M\left[y^{\prime}, y\right]$ is a shortest curve connecting $x$ and $y$ which is contained in $F$ or in $F^{\prime}$. In other words, if $x \in L$ and $y \in M$, $x, y \neq O$, are sufficiently close to $O$, then there exists a shortest curve with $[x, y] \subset F$ or $[x, y] \subset F^{\prime}$. 
We claim that $[x, y] \subset F^{\prime}$ is impossible if $x$ and $y$ are sufficiently close to $O$. For otherwise, there exists a sequence $\left(x_{n}\right)$ of points on $L$ different from $O$ with $x_{n} \rightarrow O$, a sequence $\left(y_{n}\right)$ of points on $M$ different from $O$ with $y_{n} \rightarrow O$ and a sequence of shortest curves $\left[x_{n}, y_{n}\right] \subset F^{\prime}$. This implies (cf. [3, Thm. 3, p. 22]) that $\angle_{s} F^{\prime}=\angle(L, M) \leq \pi$. But this contradicts $\angle_{s} F^{\prime}>\pi$.

Hence, if $x \in L$ and $y \in M, x, y \neq O$, are sufficiently close to $O$, then there exists a shortest curve with $[x, y] \subset F$. Now the claim follows from (4).

We also use a general isoperimetric inequality due to Aleksandrov, which we state only for the case of non-positively curved surfaces.

Proposition 3 Let $S$ be a non-positively curved simply connected Aleksandrov surface. If $\Omega \subset S$ is a Jordan region, then

$$
4 \pi A(\Omega) \leq \ell^{2}(\partial \Omega) .
$$

If $\Gamma \subset S$ is a closed curve, then

$$
4 \pi A\left(\left\{a \in S \backslash \Gamma: \operatorname{ind}_{a} \Gamma \neq 0\right\}\right) \leq \ell^{2}(\Gamma),
$$

where $\operatorname{ind}_{a} \Gamma$ denotes the index of $\Gamma$ with respect to $a$.

Proof. For the proof of the first statement, see for example [7, p. 11]. The second statement follows from the first.

A metric space is called geodesic if every two points can be connected by a shortest curve. A non-complete Aleksandrov surface need not be a geodesic space, so we will need an approximation by geodesic spaces.

Let $\Omega \subset S$ be a closed Jordan region bounded by a Jordan broken line. Then $\Omega$ is a metric space with the distance

$$
\rho_{\Omega}(x, y)=\inf \{\ell(\gamma): \gamma \subset \Omega, \gamma \text { is a curve connecting } x \text { and } y\} .
$$

A curve in $\Omega$ connecting two points $x, y \in \Omega$ whose length is equal to $\rho_{\Omega}(x, y)$ is a shortest curve with respect to the metric $\rho_{\Omega}$ and is denoted by $[x, y]_{\Omega}$. It easily follows from the Arzelá-Ascoli Theorem that for every two points $x, y \in \Omega$ a shortest curve $[x, y]_{\Omega} \subset \Omega$ exists. In other words, $\left(\Omega, \rho_{\Omega}\right)$ is a geodesic metric space. 
There exists an increasing sequence of closed Jordan regions $\left(\Omega_{k}\right)$, bounded by broken lines, that exhaust our surface $S$. We have

$$
\rho(x, y)=\lim _{k \rightarrow \infty} \rho_{\Omega_{k}}(x, y) \quad \text { for } \quad x, y \in S .
$$

Using the uniqueness of shortest curves in a non-positively curved Aleksandrov space, it is easy to see that $[x, y]_{\Omega}$ is a broken line (with respect to the original metric $\rho$ ), but we could not find an appropriate reference for this uniqueness statement. So we choose to use the following lemma instead.

Lemma 1 Let $\Omega \subset S$ be a closed Jordan region bounded by a Jordan broken line. Then for every $\epsilon>0$, all points $x, y \in \Omega$, and every shortest curve $L_{1}=[x, y]_{\Omega}$ there exists a shortest curve $L_{2}=[x, y]_{\Omega}$ such that $L_{2}$ is a broken line with respect to the original metric and the Hausdorff distance with respect to the metric $\rho_{\Omega}$ between $L_{1}$ and $L_{2}$ is less than $\epsilon$.

Proof. As we stated above in this section, every point $a$ in an Aleksandrov surface has arbitrarily small convex neighborhoods $U$. If $a \in \Omega$ and $U$ is a sufficiently small convex neighborhood of $a$, then $U$ is starlike with respect to $a$. By this we mean that for every point $x \in U \cap \Omega$ there exists a shortest curve $[a, x] \subset \Omega$.

This statement is clear for interior points of $\Omega$. If $a \in \partial \Omega$, then there are two shortest curves $L, M \subset \partial \Omega$ with $L \cap M=\{a\}$ such that $a$ is in the interior of $L \cup M$. We may assume that the convex neighborhood $U$ of $a$ does not meet $\partial \Omega \backslash(L \cup M)$. Let $K=[x, a] \subset U$ be a shortest curve with endpoints $x$ and $a$. Since it meets the boundary $\partial \Omega$, the first point $y$ on $K$ which belongs to $\partial \Omega$ has to lie on $L \cup M$. We may assume $y \in L$. Then $K[x, y] \cup L[y, a]$ is a shortest curve (in the original metric) connecting $x$ and $a$ that lies in $\Omega$.

Using these convex neighborhoods for points on $L_{1}$ and a standard covering argument, we see that there are points $a_{1}=x, a_{2}, \ldots, a_{n}=y$ on $L_{1}$, enumerated in the order of increasing parameter, and neighborhoods $U_{k}$ of $a_{k}$ that are starlike with respect to $a_{k}$, whose $\rho$-diameter is less than $\epsilon / 2$, and such that $L_{1}\left[a_{k}, a_{k+1}\right] \subset U_{k}$ for $k \in\{0, \ldots, n-1\}$.

Using the starlikeness of the sets $U_{k}$ we can find shortest curves $\left[a_{k}, a_{k+1}\right] \subset$ $\Omega$. Let $L_{2}=\left[a_{0}, a_{1}\right] \cup \ldots \cup\left[a_{n-1}, a_{n}\right] \subset \Omega$. This is a curve connecting $x$ and $y$ in $\Omega$. Moreover, $\ell\left(L_{2}\right) \leq \ell\left(L_{1}\right)$. Therefore, $\rho_{\Omega}(x, y)=\ell\left(L_{2}\right)$ and $L_{2}$ is a shortest curve in the metric $\rho_{\Omega}$, that is, $L_{2}=[x, y]_{\Omega}$. Finally, it is clear 
that the Hausdorff distance between $L_{1}$ and $L_{2}$ is less than $\epsilon$, since for every point $a \in L_{1}$ we can find a point $b \in L_{2}$ with $\rho_{\Omega}(a, b)<\epsilon$ and vice versa.

\section{The curvature condition (i) implies Gromov hyperbolicity}

In this section, $S$ is an open simply connected non-positively curved Aleksandrov surface which satisfies the condition (i) of our Theorem. Let $\Omega$ be a Jordan region in $S$ bounded by a broken line. In view of (7) it is enough to prove (ii) for the metric space $\left(\Omega, \rho_{\Omega}\right)$ with $\delta$ independent of $\Omega$.

Instead of proving the inequality (2) in (ii) for the metric $\rho_{\Omega}$ and for points $x, y, z, w \in \Omega$ directly, we verify the following property, which for geodesic metric spaces is quantitatively equivalent to (ii) ([8], Proposition 3.4 on p. 10$)$.

Proposition 4 Suppose that $\Omega \subset S$ is a closed Jordan region bounded by a Jordan broken line, and $x_{1}, x_{2}, x_{3}$ are three points in $\Omega$. Let $\left[x_{i}, x_{j}\right]_{\Omega}$ be any three shortest curves with respect to the metric $\rho_{\Omega}$ connecting these points. Then there exist points $y_{1} \in\left[x_{2}, x_{3}\right]_{\Omega}, y_{2} \in\left[x_{1}, x_{3}\right]_{\Omega}$, and $y_{3} \in\left[x_{1}, x_{2}\right]_{\Omega}$ such that

$$
\max \left\{\rho_{\Omega}\left(y_{i}, y_{j}\right): i, j \in\{1,2,3\}\right\} \leq C\left(\epsilon, R_{0}\right),
$$

where $\epsilon$ and $R_{0}$ are the constants in (i).

We emphasize that $C(\epsilon, R)$ does not depend on the choice of $\Omega$.

Proof. By Lemma 1 we may assume that the curves $\left[x_{i}, x_{j}\right]_{\Omega}$ are broken lines. We will further reduce the proof to the case when their union is a Jordan curve.

Lemma 2 Either all three curves $\left[x_{i}, x_{j}\right]_{\Omega}$ have a common point, or they have subarcs $\left[\tilde{x}_{i}, \tilde{x}_{j}\right]_{\Omega} \subset\left[x_{i}, x_{j}\right]_{\Omega}$, which form a Jordan curve if traversed in appropriate order.

Proof. For $(i, j) \in\{(1,2),(2,3),(3,1)\}$ consider the oriented $\operatorname{arcs} L_{i, j}=$ $\left[x_{i}, x_{j}\right]_{\Omega}$. Let $x_{1}^{\prime}$ be the last point on $L_{1,2}$ which belongs to the intersection 
$L_{1,2} \cap L_{3,1}$. If $x_{1}^{\prime}$ coincides with $x_{2}$ or $x_{3}$ then $x_{1}^{\prime}$ is a common point of all three $\operatorname{arcs}\left[x_{i}, x_{j}\right]_{\Omega}$. Suppose that this is not the case. Then the new triangle formed by $L_{1,2}^{\prime}:=L_{1,2}\left[x_{1}^{\prime}, x_{2}\right], L_{2,3}^{\prime}:=L_{2,3}$ and $L_{3,1}^{\prime}:=L_{3,1}\left[x_{3}, x_{1}^{\prime}\right]$, has the property that its sides $L_{1,2}^{\prime}$ and $L_{3,1}^{\prime}$ intersect only at $x_{1}^{\prime}$. Now repeat this construction, starting from $x_{2}$. Namely let $x_{2}^{\prime}$ be the last point on $L_{2,3}^{\prime}$ which belongs to $L_{1,2}^{\prime}$. If $x_{2}^{\prime}$ coincides with $x_{1}^{\prime}$ or $x_{3}$, then again $x_{2}$ is a common point of all three sides $\left[x_{i}, x_{j}\right]$. Otherwise the triangle formed by $L_{1,2}^{\prime \prime}:=L_{1,2}^{\prime}\left[x_{1}^{\prime}, x_{2}^{\prime}\right], L_{2,3}^{\prime \prime}:=L_{2,3}^{\prime}\left[x_{2}^{\prime}, x_{3}\right]$ and $L_{3,1}^{\prime \prime}:=L_{3,1}^{\prime}$ has the property that $L_{1,2}^{\prime \prime}$ intersects the other sides at one point each. If this last triangle is still not Jordan, we have to repeat the above construction once more, starting at $x_{3}$.

We continue our proof of Proposition 4, assuming that the union of the $\operatorname{arcs}\left[x_{i}, x_{j}\right]_{\Omega}$ is a Jordan broken line. We denote by $\Omega^{*}$ its interior region, and by $L_{0}, \ldots, L_{n-1}$ (labeled by residues modulo $n$, in the cyclic order induced by the orientation of $\left.\partial \Omega^{*}\right)$ a decomposition of $\partial \Omega^{*}$ into shortest segments. We assume that the points $x_{1}, x_{2}$ and $x_{3}$ are among the vertices where successive $\operatorname{arcs} L_{k}$ and $L_{k+1}$ abut. Let $F_{k}$ be the sector formed by $L_{k}$ and $L_{k+1}$, which is contained in $\overline{\Omega^{*}}$, and let $\alpha_{k}=\angle_{s} F_{k}$. Proposition 2 implies that if the vertex of $F_{k}$ is different from $x_{1}, x_{2}$ and $x_{3}$, then $\alpha_{k}$ is at least $\pi$, for otherwise our shortest lines can be shortened inside $\overline{\Omega^{*}} \subset \Omega$. Thus the Gauss-Bonnet Theorem (Proposition 1), applied to $\Omega^{*}$ yields

$$
\omega\left(\Omega^{*}\right) \geq 2 \pi-3 \pi+\beta_{1}+\beta_{2}+\beta_{3} \geq-\pi,
$$

where $\beta_{i} \geq 0$ is the sectorial angle of the sector whose vertex is $x_{i}$. Thus the integral curvature of $\Omega^{*}$ is at least $-\pi$.

So if the condition (i) is satisfied, $\Omega^{*}$ cannot contain open $\rho$-discs of radius greater than $R_{1}:=2 R_{0} \pi / \epsilon+1$. Indeed, suppose that such a disc $B$ is contained in $\Omega^{*}$. Then $B$ is relatively compact in $S$, and contains at least $\pi / \epsilon$ disjoint relatively compact open $\rho$-discs of radius $R_{0}$ in $S$. Thus the integral curvature of $B$ is less than $-\pi$, which contradicts the fact that the integral curvature of $\Omega^{*}$ is at least $-\pi$.

If an open $\rho_{\Omega}$-disc is contained in the interior of $\Omega$, then it coincides with the open $\rho$-disc with the same center and radius. From this we conclude that $\Omega^{*}$ cannot contain any open disc of radius $R_{1}$ with respect to the metric $\rho_{\Omega}$.

Thus we established the following: every point $x$ in $\Omega^{*}$ has $\rho_{\Omega}$-distance less than $R_{1}$ from one of the three sides of $\Omega^{*}$. According to a result of Sperner (cf. [4, p. 378]), if a closed 2 -simplex is covered by three open sets, 
each containing one side, then these three sets have non-empty intersection. Thus we conclude that there is a point $x_{0} \in \Omega^{*}$ which is within $\rho_{\Omega}$-distance $R_{1}$ from each of the three sides. Our assertion (8) follows with $C=2 R_{1}$.

This concludes the proof of the implication (i) $\Rightarrow$ (ii).

\section{Gromov hyperbolicity implies a linear isoperi- metric inequality}

The following lemma and proposition apply to a $K$-almost geodesic space $S$, which means that for every $x, y \in S$ and $t \in[0, \rho(x, y)]$ there exists a point $z \in S$ such that $\rho(x, z) \leq t+K$ and $\rho(y, z) \leq \rho(x, y)-t+K$. Evidently, every space with an intrinsic metric is $K$-almost geodesic for every $K>0$. We assume that $S$ satisfies (ii), and $\delta>0$ is the parameter from (ii). We fix a base point $w \in S$ and use the notation $|u|=|u|_{w}=\rho(u, w),|u-v|=\rho(u, v)$ for $u, v \in S$. Then condition (ii) can be rewritten as

$$
|x-y| \leq 2 \delta+\max \{|x-z|+|y|-|z|,|y-z|+|x|-|z|\}, \quad x, y, z \in S .
$$

Lemma 3 Suppose $S$ is a $K$-almost geodesic space, satisfying (9). Let $d \geq$ $10 \delta+2 K$, and let $x_{0}, \ldots, x_{N-1}$ be $N \geq 4$ points in $S$ labeled by residues modulo $N$ such that

$$
\left|x_{k}-x_{k+1}\right| \leq d \quad \text { for } \quad k \in\{0, \ldots, N-1\} .
$$

Then there exists $m \in\{0, \ldots, N-1\}$ such that one of the following conditions holds:

a) $\left|x_{m-1}-x_{m+1}\right| \leq d$, or

b) there exist $x \in S$ such that $\left|x_{k}-x\right| \leq d$ for $k \in\{m-2, m-1, m, m+1\}$.

Proof. Let $m \in\{0, \ldots, N-1\}$ with $\left|x_{m}\right|=\max \left\{\left|x_{0}\right|, \ldots,\left|x_{N-1}\right|\right\}$. If $\left|x_{m-1}-x_{m+1}\right| \leq d$, we are done. So we may assume $\left|x_{m-1}-x_{m+1}\right|>d$. On the other hand, by (9) with $x=x_{m-1}, y=x_{m+1}$ and $z=x_{m}$ we have

$$
\left|x_{m-1}-x_{m+1}\right| \leq d+2 \delta .
$$

The same inequality (9), together with $\left|x_{m-1}-x_{m+1}\right|>d$, gives

$$
\max \left\{\left|x_{m-1}\right|,\left|x_{m+1}\right|\right\} \geq\left|x_{m}\right|-2 \delta \text {. }
$$


We may assume without loss of generality that

$$
\left|x_{m-1}\right| \geq\left|x_{m}\right|-2 \delta \text {. }
$$

Notice that

$$
\left|x_{m-1}\right| \geq d / 2-\delta
$$

for otherwise

$$
\begin{aligned}
\left|x_{m+1}-x_{m-1}\right| & \leq\left|x_{m+1}\right|+\left|x_{m-1}\right| \leq\left|x_{m}\right|+\left|x_{m-1}\right| \\
& \leq 2\left|x_{m-1}\right|+2 \delta \leq d
\end{aligned}
$$

which contradicts our assumption.

Put $t:=4 \delta+K$. From (12) and $d \geq 10 \delta+2 K$ we obtain

$$
0 \leq t \leq d / 2-\delta \leq\left|x_{m-1}\right|
$$

therefore $t \in\left[0,\left|x_{m-1}\right|\right]$. By the definition of a $K$-almost geodesic space, there exists $x \in S$ such that

$$
\left|x-x_{m-1}\right| \leq t+K
$$

and

$$
|x| \leq\left|x_{m-1}\right|-t+K
$$

Using (9) with $y=x_{m-2}$ and $z=x_{m-1}$, inequality (11) and the definition of $t$, we obtain

$$
\left|x-x_{m-2}\right| \leq 2 \delta+\max (t+K+2 \delta, d-t+K) \leq d .
$$

Similarly

$$
\left|x-x_{m}\right| \leq 2 \delta+\max (t+K+2 \delta, d-t+K) \leq d .
$$

Using (9) with $y=x_{m+1}$ and $z=x_{m-1}$, inequality (10) and the definition of $t$, we obtain

$$
\left|x-x_{m+1}\right| \leq 2 \delta+\max (t+K+2 \delta, d+2 \delta-t+K) \leq d .
$$

Thus by (14)-(16) we have $\left|x-x_{k}\right| \leq d$ for $k \in\{m-2, m, m+1\}$. Finally (13) implies $\left|x-x_{m-1}\right| \leq 4 \delta+2 K \leq d$, and this completes the proof. 
Proposition 5 Suppose that $S$ is a $K$-almost geodesic metric space, which satisfies (9). Then for $d \geq 10 \delta+2 K$ and $N \geq 3$ the following is true.

If $v_{0}, \ldots, v_{N-1}$ are distinct points on the unit circle $\partial \mathbf{D}$ labeled by residues modulo $N$ in cyclic order, and $f:\left\{v_{0}, \ldots, v_{N-1}\right\} \rightarrow S$ is a map such that

$$
\left|f\left(v_{k}\right)-f\left(v_{k+1}\right)\right| \leq d \quad \text { for } \quad k \in\{0, \ldots, N-1\},
$$

then there exists a triangulation $T$ of the unit disc $\mathbf{D}$ by topological triangles $\Delta_{1}, \ldots, \Delta_{M}$ with the following properties:

a) $M \leq 3 N$,

b) the set of vertices of $T$ on $\partial \mathbf{D}$ coincides with $\left\{v_{0}, \ldots, v_{N-1}\right\}$,

c) there is an extension of $f$ to the set of all vertices $V$ of $T$, satisfying $|f(u)-f(v)| \leq d$, whenever the vertices $u$ and $v$ are connected by an edge in $T$.

This follows from Lemma 3 by induction on $N$ as in [8, pp. 62-64].

Now we suppose that $S$ is a surface as in our Theorem satisfying (ii). We will prove a linear isoperimetric inequality. Since $S$ is $K$-almost geodesic for every $K>0$ we can choose $K=1$ and $d=10 \delta+2$ in Proposition 5. Let $\Omega \subset S$ be a Jordan region, and assume that $\ell(\partial \Omega)<\infty$ (otherwise there is nothing to prove). We consider two cases.

Case 1. $\ell(\partial \Omega) \leq 2 d$. Then by Proposition 3

$$
A(\Omega) \leq \frac{1}{4 \pi} \ell^{2}(\partial \Omega) \leq \frac{d}{2 \pi} \ell(\partial \Omega)=C_{1} \ell(\partial \Omega) .
$$

Case 2. $\ell(\partial \Omega)>2 d$. Consider a parameterization $f: \partial \mathbf{D} \rightarrow \partial \Omega$. Then we can find distinct points $v_{0}, \ldots, v_{N-1} \in \partial \mathbf{D}$ in counterclockwise cyclic order on $\partial \mathbf{D}$ labeled by residues modulo $N$ such that

$$
\ell\left(f\left(\left[v_{k}, v_{k+1}\right]\right)\right) \leq d \quad \text { for } \quad k \in\{0, \ldots, N-1\},
$$

where $\left[v_{k}, v_{k+1}\right]$ is the subarc of $\partial \mathbf{D}$ from $v_{k}$ to $v_{k+1}$, and

$$
3 \leq N \leq \frac{\ell(\partial \Omega)}{d}+1 \leq \frac{2}{d} \ell(\partial \Omega) .
$$

Proposition 5 provides a triangulation $T$ of $\mathbf{D}$ with the set of vertices $V$ and triangles $\Delta_{1}, \ldots, \Delta_{M}$ and an extension of $f$ to $V$ with the properties a), b) 
and c). We choose an orientation of the triangulation consistent with the cyclic order of the vertices on $\partial \Omega$. If $e$ is an oriented edge of the triangulation $T$ with endpoints $w_{1}$ and $w_{2}$, we choose an arc $\beta(e)$ connecting $f\left(w_{1}\right)$ and $f\left(w_{2}\right)$ whose length is at most $d+1$. This is possible in view of $\mathrm{c}$ ). For edges that differ only by their orientation we choose the same arcs traversed in opposite directions. For every oriented triangle $\Delta_{k}$ of $T$ whose oriented boundary consists of $e_{k}^{1}, e_{k}^{2}, e_{k}^{3}$ we define a closed curve

$$
\alpha_{k}=\beta\left(e_{k}^{1}\right) \cup \beta\left(e_{k}^{2}\right) \cup \beta\left(e_{k}^{3}\right) .
$$

Here it is understood that the curves $\beta\left(e_{k}^{i}\right)$ are traversed according to the orientation of the edges $e_{k}^{i}$. In the same way we define a closed curve

$$
\alpha=\bigcup_{k=0}^{N-1} \beta\left(e\left(v_{k}, v_{k+1}\right)\right),
$$

where $e\left(v_{k}, v_{k+1}\right)$ stands for the oriented edge of $T$ with vertices $v_{k}$ and $v_{k+1}$.

From the definition of these curves based on the triangulation $T$ it follows that for all points $w \in S$ that do not lie on $\alpha$ or any of the curves $\alpha_{k}$ we have

$$
\operatorname{ind}_{w} \alpha=\sum_{k=1}^{M} \operatorname{ind}_{w} \alpha_{k} .
$$

Using the isoperimetric inequality (6) in Proposition 3 and a) from Proposition 5 , we obtain

$$
\begin{aligned}
A\left(\left\{w \in S \backslash \alpha: \operatorname{ind}_{w} \alpha \neq 0\right\}\right) & \leq \bigcup_{k=1}^{M} A\left(\left\{w \in S \backslash \alpha_{k}: \operatorname{ind}_{w} \alpha_{k} \neq 0\right\}\right) \\
& \leq \frac{1}{4 \pi} \sum_{k=1}^{M} \ell^{2}\left(\alpha_{k}\right) \leq \frac{9(d+1)^{2}}{4 \pi} M \\
& \leq \frac{27(d+1)^{2}}{4 \pi} N \leq c_{1}(d) \ell(\partial \Omega) .
\end{aligned}
$$

We can find an at most countable set of subarcs with disjoint interiors $\gamma_{k} \subset$ $\partial \Omega$ and subarcs with disjoint interiors $\beta_{k} \subset \alpha$ such that

a) $\gamma_{k} \cup \beta_{k}$ are Jordan curves for $k \in \mathbf{N}$,

b) If $w \in S \backslash(\partial \Omega \cup \alpha)$ and $\operatorname{ind}_{w} \partial \Omega \neq \operatorname{ind}_{w} \alpha$, then $w$ belongs to the interior region of one of the Jordan curves $\gamma_{k} \cup \beta_{k}$, 
c) $\sup _{k \in \mathbf{N}}\left\{\ell\left(\gamma_{k}\right), \ell\left(\beta_{k}\right)\right\} \leq d+1 \leq 2 d$.

Hence

$$
\begin{aligned}
& A\left(\left\{w \in S \backslash(\partial \Omega \cup \alpha): \operatorname{ind}_{w} \partial \Omega \neq \operatorname{ind}_{w} \alpha\right\}\right) \leq \frac{1}{4 \pi} \sum_{k=1}^{\infty}\left(\ell\left(\gamma_{k}\right)+\ell\left(\beta_{k}\right)\right)^{2} \\
\leq & \frac{1}{2 \pi} \sum_{k=1}^{\infty}\left(\ell^{2}\left(\gamma_{k}\right)+\ell^{2}\left(\beta_{k}\right)\right) \leq \frac{d}{\pi} \sum_{k=1}^{\infty}\left(\ell\left(\gamma_{k}\right)+\ell\left(\beta_{k}\right)\right) \leq \frac{d}{\pi}(\ell(\partial \Omega)+\ell(\alpha)) \\
\leq & \left.\frac{d}{\pi}(\ell(\partial \Omega)+2 d N)\right) \leq \frac{5 d}{\pi} \ell(\partial \Omega)=c_{2}(d) \ell(\partial \Omega) .
\end{aligned}
$$

Combining the last two estimates we finally obtain

$$
\begin{aligned}
A(\Omega) & =A\left(\left\{w \in S \backslash \partial \Omega: \operatorname{ind}_{w} \partial \Omega=1\right\}\right) \\
& \leq A\left(\left\{w \in S \backslash \alpha: \operatorname{ind}_{w} \alpha \neq 0\right\}\right)+c_{2}(d) \ell(\partial \Omega) \\
& \leq\left(c_{1}(d)+c_{2}(d)\right) \ell(\partial \Omega)=: C_{1}(d) \ell(\partial \Omega) .
\end{aligned}
$$

\section{A linear isoperimetric inequality implies tight- ness}

To show that $S$ is tight it is enough to give an upper bound for $\left\|f^{\prime}(0)\right\|$ whenever $f: \mathbf{D} \rightarrow S$ is an injective conformal map. The bound for other points and other holomorphic maps will then follow from Schwarz's Lemma.

Let $f: \mathbf{D} \rightarrow S$ be a conformal map. Then the images $f(D(t))$ of the discs $D(t)=\{z:|z|<t\}$ for $t \in(0,1)$ are Jordan regions. Let $A(t)=A(f(D(t)))$ and $\ell(t)=\ell(f(D(t))), t \in(0,1)$. Then by assumption we have

$$
A(t) \leq C_{1} \ell(t), \quad 0<t<1 .
$$

For $r \in(0,1)$ we consider the family of curves $\Gamma_{r}$ consisting of all circles $\{z \in \mathbf{D}:|z|=t\}$ with $t \in[r, 1)$. Then for the conformal modulus of this curve family (cf. [1]) we have $\bmod \Gamma_{r}=(2 \pi)^{-1} \log (1 / r)$ for $r \in(0,1)$. On the other hand, the density

$$
\lambda(z):=\frac{1-|z|^{2}}{2 \ell(|z|)}\left\|f^{\prime}(z)\right\|, \quad r \leq|z|<1,
$$


is admissible for $\Gamma_{r}$. Hence

$$
\begin{aligned}
\bmod \Gamma_{r} & \leq \int_{r \leq|z|<1}\left\|f^{\prime}(z)\right\|^{2} \frac{\left(1-|z|^{2}\right)^{2}}{4 \ell^{2}(|z|)} d m(z)=\int_{r}^{1} \frac{d A(t)}{\ell^{2}(t)} \\
& \leq C_{1}^{2} \int_{r}^{1} \frac{d A(t)}{A^{2}(t)} \leq \frac{C_{1}^{2}}{A(r)} .
\end{aligned}
$$

Therefore

$$
A(r) \leq \frac{2 \pi C_{1}^{2}}{\log (1 / r)}, \quad 0<r<1 .
$$

On the other hand, by Proposition 3

$$
\begin{aligned}
A(t) & \leq \frac{1}{4 \pi} \ell^{2}(t)=\frac{1}{4 \pi}\left[\int_{0}^{2 \pi} \frac{1-t^{2}}{2}\left\|f^{\prime}\left(t e^{i \theta}\right)\right\| t d \theta\right]^{2} \\
& \leq \frac{t}{2} \int_{0}^{2 \pi} \frac{\left(1-t^{2}\right)^{2}}{4}\left\|f^{\prime}\left(t e^{i \theta}\right)\right\|^{2} t d \theta=\frac{t}{2} A^{\prime}(t) .
\end{aligned}
$$

Thus for $0<t_{1} \leq t_{2}<1$

$$
\int_{t_{1}}^{t_{2}} \frac{2}{t} d t=2 \log \left(t_{2} / t_{1}\right) \leq \int_{t_{1}}^{t_{2}} \frac{A^{\prime}(t)}{A(t)} d t=\log \left(A\left(t_{2}\right) / A\left(t_{1}\right)\right),
$$

which implies $A\left(t_{1}\right) / t_{1}^{2} \leq A\left(t_{2}\right) / t_{2}^{2}$. Now we obtain from (20)

$$
\left\|f^{\prime}(0)\right\|^{2}=\lim _{t \rightarrow 0} \frac{4 A(t)}{\pi t^{2}} \leq \frac{16}{\pi} A(1 / 2) \leq \frac{32 C_{1}^{2}}{\log 2} .
$$

This completes the proof of the implication (iii) $\Rightarrow$ (iv).

\section{Tightness implies the curvature condition (i)}

In this section we denote the total variation of the integral curvature by $|\omega|$, and let $\bar{B}(a, r):=\{x \in S: \rho(a, x) \leq R\}$ for $a \in S, R>0$. We first need several lemmas about discs and circles on our Aleksandrov surface $S$.

Lemma 4 Every relatively compact open metric disc $B$ in $S$ is a Jordan region.

A more general result of Burago and Stratilatova [6, Theorem 2] shows that this is true if $\omega^{+}(S)<\pi$. 
Lemma 5 Suppose a disc $B(a, R)$, where $a \in S$ and $R>0$, is relatively compact in $S$ and $|\omega|(B(a, R)) \leq K_{0}$. Then

$$
A(B(a, R)) \leq C\left(K_{0}\right) R^{2},
$$

where $C\left(K_{0}\right)$ depends only on $K_{0}$.

Proof. The discs $B(a, r), r>0$, are Jordan region by the previous lemma. An estimate proved by Burago and Stratilatova [6, p. 120] shows that for $0<r<R$

$$
\ell(\partial B(a, r)) \leq r(2 \pi+|\omega|(\bar{B}(a, r))) .
$$

Combining this with the isoperimetric inequality in Proposition 3, we obtain

$$
A(B(a, r)) \leq \frac{1}{4 \pi} \ell^{2}(\partial B(a, r)) \leq \frac{1}{4 \pi}(2 \pi+|\omega|(\bar{B}(a, r)))^{2} r^{2} .
$$

Letting $r$ tend to $R$ the lemma follows.

Actually, for non-positively curved surfaces one can prove the sharp estimate

$$
A(B(a, R)) \leq(\pi+|\omega|(B(a, R)) / 2) R^{2},
$$

but we do not need this result.

Lemma 6 Let $a \in S$. Suppose that the discs $B(a, r)$ are relatively compact and that $A(B(a, r)) \leq K r^{2}$ for $r \in(0, R]$, where $R>0$ and $K>0$. Let $\Gamma$ be the family of all curves connecting $\bar{B}\left(a, R_{1}\right)$ with $S \backslash B\left(a, R_{2}\right)$, where $0<2 R_{1} \leq R_{2} \leq R$. Then

$$
\bmod \Gamma \leq \frac{16 K}{\log \left(R_{2} / R_{1}\right)} .
$$

Proof. We define a Borel density $\lambda$ on $S$ by

$$
\lambda(x)=\left\{\begin{array}{cl}
\frac{1}{\rho(x, a) \log \left(R_{2} / R_{1}\right)} & \text { for } x \in B\left(a, R_{2}\right) \backslash B\left(a, R_{1}\right), \\
0 & \text { elsewhere. }
\end{array}\right.
$$

Then $\lambda$ is admissible for $\Gamma$ because for every $\gamma \in \Gamma$ we have

$$
\int_{\gamma} \lambda(x)|d x| \geq \frac{1}{\log \left(R_{2} / R_{1}\right)} \int_{R_{1}}^{R_{2}} \frac{d s}{s}=1 .
$$


Let $N$ be the integer such that $R_{2}^{-(N+1)}<R_{1} \leq R_{2} 2^{-N}$. Then $N \leq$ $(\log 2)^{-1} \log \left(R_{2} / R_{1}\right)$. Using the notation $B_{k}=B\left(a, 2^{-k} R_{2}\right), k \in\{0, \ldots, N+$ $1\}$, we obtain

$$
\begin{aligned}
\bmod \Gamma & \leq \int_{S} \lambda^{2} d A=\int_{B\left(a, R_{2}\right) \backslash B\left(a, R_{1}\right)} \lambda^{2} d A \\
& \leq \sum_{k=0}^{N} \int_{B_{k} \backslash B_{k+1}} \lambda^{2} d A \leq \frac{1}{\log ^{2}\left(R_{2} / R_{1}\right)} \sum_{k=0}^{N} \frac{A\left(B_{k}\right)}{\left(R_{2} 2^{-(k+1)}\right)^{2}} \\
& \leq \frac{4(N+1) K}{\log ^{2}\left(R_{2} / R_{1}\right)} \leq \frac{8 N K}{\log ^{2}\left(R_{2} / R_{1}\right)} \leq \frac{16 K}{\log \left(R_{2} / R_{1}\right)}
\end{aligned}
$$

Now the proof of the implication (iv) $\Rightarrow$ (i) and of the Theorem is concluded by

Proposition 6 Let $B(a, R)$ be a disc in an Aleksandrov surface which is a Jordan region, and

$$
|\omega|(B(a, R)) \leq 1 .
$$

If $f: \mathbf{D} \rightarrow B(a, R), f(0)=a$, is a conformal homeomorphism, then

$$
R \leq C \max _{|z|<1 / 2}\left\|f^{\prime}(z)\right\|,
$$

where $C>0$ is a universal constant.

Proof. Lemmas 5 and 6 imply the existence of a universal constant $c \in$ $(0,1 / 2]$ such that

$$
\bmod \Gamma_{1}<\frac{2 \pi}{\log 2},
$$

whenever $B(a, R)$ satisfies $(21)$ and $\Gamma_{1}$ is the family of curves in $B(a, R)$ connecting $\bar{B}(a, c R)$ to $S \backslash B(a, R)$. Since $B(a, R)$ is a Jordan region, we have $\lim _{|z| \rightarrow 1} \rho(f(z), a)=R$.

Let us show that $B(a, c R)$ does not contain $f(D(1 / 2))$. Suppose that it does. Let $\Gamma_{2}$ be the family of all curves in $\mathbf{D}$, connecting $\bar{D}(1 / 2)$ with $\partial \mathbf{D}$. Then the curves in the family $f\left(\Gamma_{2}\right):=\left\{f \circ \gamma: \gamma \in \Gamma_{2}\right\}$ connect $\bar{B}(a, c R)$ with $S \backslash B(a, R)$. Thus by the conformal invariance of the modulus we have

$$
\frac{2 \pi}{\log (1 / 2)}=\bmod \Gamma_{2}=\bmod f\left(\Gamma_{2}\right) \leq \bmod \Gamma_{1}<\frac{2 \pi}{\log (1 / 2)},
$$


a contradiction. So there exists $w \in D(1 / 2)$ with the property $\rho(f(w), f(a)) \geq$ $c R$. Hence

$$
\begin{aligned}
c R & \leq \rho(f(w), f(a)) \leq \frac{1}{2} \max _{|z|<1 / 2} \frac{2}{1-|z|^{2}}\left\|f^{\prime}(z)\right\| \\
& \leq \frac{4}{3} \max _{|z|<1 / 2}\left\|f^{\prime}(z)\right\| .
\end{aligned}
$$

Acknowledgment. This research was completed while the first author was visiting Purdue University in October 1999. He thanks the people in the Department of Mathematics for their hospitality. Both authors thank K./ Astala, B./ Kleiner F./ Nazarov and A./ Volberg for discussion of this paper.

\section{References}

[1] L. Ahlfors, Conformal Invariants, McGraw-Hill, New York, 1973.

[2] L. Ahlfors, Zur Theorie der Überlagerungsflächen, Acta Math. 65 (1935), 157-194.

[3] A.D. Aleksandrov, V.A. Zalgaller, Intrinsic Geometry of Surfaces, AMS Transl. Math. Monographs, v. 15, Providence, RI, 1967.

[4] P.S. Alexandroff and H. Hopf, Topologie I, Springer, Berlin, 1935.

[5] A. Bloch, Les théorèmes de M. Valiron sur les fonctions entières et la théorie de l'uniformisation, Ann. Fac. Sci. Univ. Toulouse (3) 17 (1926), $1-22$.

[6] Yu.D. Burago and M.B. Stratilatova, A circle on a surface, Proc. Steklov Inst. Math., 76 (1967), 109-141.

[7] Yu.D. Burago and V.A. Zalgaller, Geometric Inequalities, Springer, Berlin, 1988.

[8] M. Coornaert, T. Delzant and A. Papadopoulos, Géométrie et théorie des groups, LNM, v. 1441, Springer, Berlin, 1990. 
[9] J. Dufresnoy, Sur les domains couvertes par les valeurs d'une fonction méromorphe ou algebroïde, Ann. Sci. École Norm. Sup. 58 (1941), 170259.

[10] M. Gromov, Hyperbolic groups, in: S.M. Gersten (Ed.), Essays in Group Theory, MSRI Publ. 8, Springer, New York, 1987, pp. 75-263.

[11] W. Hayman, Meromorphic Functions, Clarendon Press, Oxford, 1964.

[12] A. Huber, Zum potentialtheoretischen Aspekt der Alexandrowschen Flächentheorie, Comment. Math. Helv., 34, (1960) 99-126.

[13] S. Kobayashi, Hyperbolic Complex Spaces, Springer, Berlin, 1998.

[14] Ch. Pommerenke, Boundary Behavior of Conformal Maps, Springer, Berlin, 1992.

[15] Ch. Pommerenke, Univalent Functions, Vandenhoeck \& Ruprecht, Göttingen, 1975.

[16] Yu.G. Reshetnyak, Two-Dimensional Manifolds of Bounded Curvature, in: Yu.G. Reshetnyak (Ed.), Geometry IV. Encyclopaedia of Mathematical Sciences, v. 70, Springer, Berlin, 1993, 3-163.

Purdue University, West Lafayette, Indiana 47907, U.S.A

M.Bonk@tu-bs.de

eremenko@math.purdue.edu 